\title{
Tubular duplication of the esophagus
}

INSERM

\section{Source}

INSERM. (1999). Orphanet: an online rare disease and orphan drug data base. Tubular duplication of the esophagus. ORPHA:100048

Tubular duplication of the esophagous is a rare congenital malformation where a second structure with individual lumen and stratified squamous mucosa and muscularis mucosa lies within or adjacent to the true esophagus causing dysphagia, nausea, vomiting, retrosternal pain and respiratory problems (stridor and recurrent pneumonia) and usually presenting in children. 